Aus der psychiatrischen Universitätsklinik zu Straßburg i. E. (Direktor: Prof. Wollenberg) und aus der chirurgischen Universitätsklinik zu Straßburg i. E. (Direktor: Prof. Ma delung).

\title{
Über einen Fall von operiertem Tumor der Cauda equina').
}

Von Prof. Dr. Rosenfeld und Privatdozent Dr. Guleke.

Die Symptome und der Verlauf unseres Falles sind nicht so besonders geartet, daß eine genauere Mitteilung des Falles von neurologischer Seite erforderlich erscheint. Mitteilenswert ist der Fall nur mit Rücksicht auf den Erfolg des chirurgischen Eingriffs. Trotzdem lohnt es sich wohl der Mühe kurz darauf hinzuweisen, bis zu welchem Grade von Genauigkeit in diesem Falle die Diagnose zu stellen war, und welche der bekannten diagnostischen Grundsätze sich bewährt haben. Denn alle Beobachter weisen auf die oft noch unüberwindlichen Schwierigkeiten hin, welche entstehen, wenn es sich darum handelt die Symptome der Conus-Erkrankung von denjenigen der Caudaaffektionen scharf abzutrennen, oder wenn entschieden werden soll, ob die Cauda in ihren oberen oder unteren Partien betroffen ist. Ich setze die vielfach erörterte Symptomatologie der Caudatumoren als bekannt voraus, und bringe unseren Fall gleich zu diesen bekannten Erfahrungen in Beziehung, ohne die Literatur im einzelnen heranzuziehen.

Es handelt sich um die 46 jährige Frau Christine E., welche wegen heftiger Schmerzen im Kreuzbein zunächst in die hiesige Frauenklinik aufgenommen war. Es wurde dort nur ein Uterusmyom festgestellt, welches die Beschwerden der Kranken nicht erklären konnte. Da die Frau vollständig unfähig war zu gehen, oft unter sich ließ und in ihrem psychischen Verhalten auffällig war, wurde sie am 8. IX. Igog in die psychiatrische Klinik verlegt. Die wichtigsten Daten aus der Anam-

1) Demonstriert in der Sitzung des Unterelsässischen Arrztevereins am 3०. X. 1909 . 
nese lauten: Die Frau hatte nicht geboren und war bis vor Io Jahren gesund. Vor I5 Jahren soll sie einen Fall auf das Gesäß getan haben, welcher jedoch keine weiteren Folgen hatte. Seit Io Jahren klagt sie über Schmerzen im Kreuzbein und in den unteren Lendenwirbeln. Die Schmerzen traten anfallsweise auf und nahmen namentlich bei Bewegungen zu. Die Frau machte die Angabe, daß die Schmerzen zuerst mehr auf der rechten Seite bestanden haben und in das rechte Bein ausstrahlten. Seit etwa 5 Jahren gesellte sich eine Gangstörung hinzu. Die Beine wurden der Frau steif, namentlich gleich nach dem Aufstehen vom Stuhl oder aus der Ruhelage. Beim Sitzen waren die Schmerzen am stärksten. Seit 3 Jahren bestanden Parästhesien in der Vorderseite der Oberschenkel. Seit I $1 / 2$ Jahren war die Frau nicht mehr imstande Urin und Stuhlgang willkürlich zurückzuhalten. Sie ließ stets unter sich. Die Schmerzen hatten sich im letzten Jahre bis zur Unträglichkeit gesteigert.

Die Untersuchung am 8. IX. Igog ergab Folgendes:

Der allgemeine Eernährungszustand war noch gut. An den inneren Organen war nichts Abnormes nachweisbar. Im Urin fanden sich keine abnormen Bestandteile. Die Hirnnerven zeigten keine Funktionsstörungen. Die Bewegungsfähigkeit der oberen Extremitäten war in keiner Weise eingeschränkt. Coordinationsstörungen fehlten.

Die Kranke konnte nicht gehen; sie lag dauernd im Bett oder kniete im Bett, indem sie die Füße unter das Gesäß gelegt hatte. In dieser Stellung hatte sie angeblich am wenigsten Schmerzen. Im Liegen konnte sie mit den Beinen alle Bewegungen gut ausführen. Mit Unterstützung konnte sie einige Schritte machen. Obwohl bei passiven Bewegungen leichte Spannungszustände in den Muskeln der Oberschenkel zu erzeugen waren, so hatte der Gang doch absolut nichts Spastisches. Wenn man die Kranke nicht hielt, so fiel sie zusammen, konnte sich dann aber in der Knieellenbogenlage noch ganz gut fortbewegen. Blase und Mastdarm zeigten eine vollständige Lähmung. Beide waren dem Willen vollkommen entzogen. Die Entleerung trat mehrmals am Tage ein. Urinretention bestand nicht. Katheterisieren war niemals notwendig. Die Entleerung von Blase und Mastdarm wurde von der Kranken gefühlt. Von den spinalen Reflexen fehlte der Analreflex und der Achillessehnenreflex beiderseits. Alle übrigen Reflexe waren vorhanden. Die Kniescheibenreflexe waren lebhaft, jedoch nicht pathologisch verändert. Dorsalflexion der großen Zehe bestand nicht. Trophische Störungen ließen sich an den unteren Extremitäten nicht nachweisen. Die Muskulatur des rechten Beines war etwas magerer als diejenige des linken Beines. Muskelatrophien fehlten. Eine elektrische Untersuchung der Muskeln der unteren Extremitäten ließ die Frau nicht vornehmen. Von Sensibilitätsstörung ließ sich nur eine Reithosenanästhesie von geringer Ausdehnung nachweisen. Eine dissociierte Sensibilitätsstörung war nicht nachweisbar. In der Hautpartie, welche 
von der Sensibilitätsstörung betroffen war, fanden sich eine Anzahl roter Flecken an Stellen, welche früher von kleinen Hautdefekten eingenommen gewesen sein sollen. Die Ischiadici waren auf Druck nicht schmerzhaft; es bestand kein Ischiasphänomen. An den Unterschenkeln und Füßen waren keine anästhetischen Zonen aufzufinden. In den psychischen Verhalten der Kranken waren nur folgende Symptome auffällig: Die Kranke war sehr unruhig, änderte fortwährend ihre Lage im Bett, jammerte laut wegen der heftigen Schmerzen und zeigte lebhafte Ausdrucksbewegungen. Alle psychischen Symptome ließen sich als Reaktionen auf die heftigen Schmerzen deuten.

Man sieht, daß die exzessive Schmerzhaftigkeit, welche fast von allen Beobachtern bei den Tumoren der Cauda equina konstatiert worden ist, auch in diesem Falle das hervorstechendste Symptom gewesen ist. Das neuralgische Vorstadium dauerte mehr als Io Jahre. Die Intensität der Schmerzen wechselte anfangs. In den ersten Jahren strahlten die Schmerzen im Kreuz und in der Lendengegend nur in das rechte Bein aus. Erst einige Zeit später wurden auch im linken Bein schmerzhafte Sensationen empfunden. Diese Halbseitigkeit der Neuralgien im Anfangsstadium der Erkrankung ist vielfach als diagnostisch wichtig gegenüber den Conuserkrankungen hervorgehoben worden. Ferner bot unsere Kranke auch die oftmals beschriebene Eigentümlichkeit, daß sie, veranlaßt durch die heftigen Schmerzen, besondere Stellungen im Bett einnahm, in welchen die Schmerzen angeblich weniger intensiv waren. Sie $s a ß$ meistens im Bett, indem sie die Füße bzw. Unterschenkel unter das Gesäß gelegt hatte. Von anderen Autoren ist in derartigen Fällen die Knieellenbogenlage beobachtet worden, Obwohl die Schmerzen in das Gebiet der Ischiadici ausstrahlten, waren die großen Nervenstämme doch nicht druckempfindlich und das Ischiasphänomen fehlte. Schließlich war für die genauere Diagnose noch bedeutungsvoll, daß während der letzten 3 Jahre Parästhesien (nicht Schmerzen) im Gebiete des Nervus Cruralis dauernd bestanden.

Gegenüber diesen Neuralgien traten die Ausfallserscheinungen auf sensiblem Gebiet ganz zurück. Auch dieses ist typisch für eine reine Erkrankung der Cauda equina. Es ließ sich nur eine Reithosenanästhesie von geringer Ausdehnung nachweisen, die zur Zeit, als der Fall zum ersten Mal untersucht wurde, bereits auf beiden Seiten gleichmäßig nachweisbar war. Die Ausdehnung 
der Sensibilitätsstörung gestattete zunächst vielleicht noch den Schluß, daß der Tumor die Cauda etwa in der Höhe des 4. und 5. Lumbalwirbels komprimierte. Daß die Ausbreitung der Sensibilitätsstörung für eine genauere Lokalisation der Erkrankung in der Cauda equina im allgemeinen nicht verwertbar ist, ist bekannt. In unserem Falle war es aber doch auffällig, daß nach ro jähriger Dauer der Erkrankung die sensiblen Ausfallserscheinungen noch nicht größere geworden waren, ein Umstand, der für die Annahme einer sehr tiefsitzenden Erkrankung in der Cauda verwertet werden konnte. Die Sensibilitätsstörungen waren also in unserm Falle so geartet, wie sie gewöhnlich als charakteristisch für die Tumoren dieser Gegend angegeben werden. Auch die Art der motorischen Störungen in unserem Falle steht im Einklang mit dem, was man häufig bei tiefsitzenden Erkrankungen der Cauda equina beobachtet hat, d. h. es fanden sich keine atrophische Lähmungen in einzelnen Muskelgruppen. Die Muskeln des rechten Oberschenkels waren nur wenig in ihrem Volumen reduziert gegenüber denjenigen der anderen Seite. Im Liegen konnte die Frau alle Bewegungen gut ausführen; mit Unterstützung konnte sie an manchen Tagen einige Schritte gehen. Die Geringfügigkeit der motorischen Ausfallssymptome, wenn wir von dem Unvermögen $\mathrm{zu}$ gehen und $\mathrm{zu}$ stehen absehen, wird immer als diagnostisch wichtig für eine reine Caudaequinaläsion hervorgehoben. Uber das Verhalten der Reflexe (Fehlen der Achillessehnenreflexe und des Analreflexes) und die Blasen-Mastdarm-Störungen ist nichts Besonderes zu sagen. Die geringe Bedeutung dieser schweren Ausfallssymptome für die Differentialdiagnose zwischen Konusund Caudaerkrankung ist bekannt. Nur auf eins ist vielleicht noch hinzuweisen. Da öfters konstatiert worden ist, daß bei hochsitzenden Tumoren der Cauda equina Blase und Mastdarm besonders frühzeitig außer Funktion gesetzt werden, und in unserem Falle das Gegenteil zutraf, d. h. die Blasenstörung erst 8 Jahre nach dem Bestehen des neuralgischen Vorstadiums auftrat, so wird man diese Kombination der Symptome für die Diagnose eines tiefsitzenden Tumors der Cauda verwerten können. Schließlich ist auf den ungemein protrahierten Verlauf des Falles und auf die langsame Aufeinanderfolge der einzelnen Symptomengruppen hinzuweisen. 
Symptome und Verlauf unseres Falles wiesen auf einen ausschlieBlichen Sitz der Erkrankung in der Cauda equina hin. Ferner sprachen mancherlei Umstände dafür, daß die Cauda in ihren unteren Abschnitten von einem Tumor komprimiert wurde. Nach dieser Richtung waren folgende Symptome besonders verwertbar: Die Geringfügigkeit der definitiven motorischen und sensiblen Ausfallserscheinungen bei der langen Dauer des neuralgischen Vorstadiums und ferner das verspätete Auftreten der Blase- und Mastdarmstörung. Außerdem war durch die Rektaluntersuchung erwiesen, daß die Geschwulstbildung bis zum 2. Sakralloch reichte. Aber andererseits war die große Schmerzhaftigkeit des 5. Lendenwirbels auffällig und ferner konnte nicht unberücksichtigt bleiben, daß zu den Neuralgien im Ischiadikusgebiet während der letzten 3 Jahre starke Parästhesien auch im Gebiete des Crualis aufgetreten waren. Da also die Möglichkeit vorlag, daß der Tumor im Laufe der letzten Jahre die Tendenz hatte, sich mehr nach oben zu entwickeln, so erschien es ratsam, etwa am 3. Lendenwirbel einzugehen, um ganz sicher die obere Grenze der Erkrankung zu erreichen. Die Kranke wurde am I9. September Igog in die Chirurgische Klinik verlegt.

Während bei der Fragestellung des Neurologen sich das Hauptinteresse dahin konzentriert, den Sitz eines Rückenmarkstumors mit Hilfe der so exakt ausgebildeten Segmentdiagnose so genau wie möglich zu präzisieren, muß der Chirurg in derartigen Fällen festzustellen suchen, ob die Verhältnisse so liegen, da $B$ eine Operation indiziert erscheint oder nicht. In erster Linie ist dabei zu entscheiden, ob ein primärer oder ein metastatischer Tumor vorliegt, da man in letzterem Falle sich wohl nur in ganz seltenen Ausnahmefällen zu einem operativen Eingreifen entschließen wird.

Bei unserer Patientin lagen die Verhältnisse in dieser Beziehung nicht ganz einfach. Die Patientin bot nämlich, abgesehen von dem bereits besprochenen Nervenstatus, folgenden Befund: Die Wirbelsäule wies im Bereich der Lendenwirbel eine stärkere Lordose auf, als der Norm entspricht, und war in dieser Stellung fixiert. Der Dornfortsatz des 5. Lendenwirbels schien etwas unter das Niveau der übrigen Dornfortsätze eingesunken zu sein und war 
sehr druckempfindlich. In dieser Gegend war ein Tumor, Fluktuation oder Ödem nicht nachweisbar. Dagegen fühlte man rektal unter der intakten verschieblichen Schleimhaut, entsprechend dem rechten I. Intervertebralloch des Kreuzbeins eine etwa Io Pfennigstückgroße, glatte, ziemlich harte, gewölbte, nicht fluktuierende Prominenz, die anscheinend aus diesem Intervertebralloch herausgewachsen war. Diese Prominenz war so druckempfindlich, daß Pat. bei jeder leichten Berührung derselben laut aufschrie. In der nächsten Umgebung des Intervertebralloches schien der Knochen weniger glatt und eben, als auf der linken Seite, wo nichts Abnormes nachweisbar war.

Dieser Befund legte die Vermutung nahe, daß der auf Grund des Nervenstatus allein nicht ganz präzise auf einen bestimmten Wirbel zu lokalisierende Tumor aller Wahrscheinlichkeit nach im Bereich des 5. Lendenwirbels sitzen und von hier durch das I. rechte Intervertebralloch nach dem Becken $\mathrm{zu}$ durch das Kreuzbein hindurchgewachsen sein müsse. Die auf die untersten Lendenwirbel beschränkte Versteifung der Wirbelsäule, besonders aber die exquisite Druckschmerzhaftigkeit des 5. Lendenwirbels sprach, wie bei einer Reihe älterer, von anderen Autoren beschriebenen Fälle, mit großer Wahrscheinlickheit für diesen Sitz. Das Hindurchwachsen des Tumors durch das Intervertebralloch und die Rauhigkeit der Umrandung des letzteren ließen einen malignen Tumor annehmen, der zum Teil wohl schon den Knochen usuriert und durchwachsen hatte.

Die Hoffnung, durch das Röntgenbild genaueren Aufschluß über Sitz, Art und Größe des Tumors zu erhalten, erwies sich als trügerisch. Die Lendenwirbelsäule sah völlig normal aus, und ein von dem Kreuzbein angedeuteter leichter Schatten ließ sich für die Diagnose nicht verwerten, da Pat. Trägerin von einem wallnuB- und einem kindskopfgroßen Uterusmyom ist, die diesen Schatten sehr wohl bedingen konnten.

Die Anwesenheit der Myome machte, nun bezüglich der Indikationsstellung zur Operation einige Schwierigkeiten. Da nach den bisherigen Erfahrungen ein nicht geringer Teil der in dieser Gegend beobachteten Tumoren metastatischer Natur war, so lag die Befürchtung nahe, daß auch in unserem Falle der Tumor eine Metastase sein könnte, deren Ursprung vielleicht in einer 
sarkomatösen Degeneration einer der Uterusmyome $\mathrm{zu}$ suchen war, da sich sonstige primäre Herde nicht fanden. Der Befund der Frauenklinik (Dr. S c h i c k ele), in der Pat. untersucht war, sprach indessen gegen diese Annahme, des weiteren aber vor allem die Anamnese, die mit Bestimmtheit den Beginn der spinalen Erkrankung auf mindestens Io Jahre zurückverlegen ließ. Daß ein metastatischer Tumor an dieser Stelle sich so langsam entwickeln sollte, ohne daß sonstige Metastasen in der Zwischenzeit aufgetreten wären und ohne daß der primäre Herd jetzt deutlich nachweisbar wäre, das ließ sich mit ziemlicher Sicherheit ausschließen.

So nahm ich am 25. IX. Igog die Operation in Bauchlage der Pat. mit erhöhtem Becken vor. Da der Sitz des Tumors nicht ganz sicher angegeben werden konnte, so resezierte ich von vornherein den 3 . bis 5. Lendenwirbelbogen, und legte die Dura in dieser Ausdehnung frei, um nicht genötigt zu sein, etwa nach Eröffnung der Dura noch weitere Resektionen vornehmen zu müssen. Die Dura lie $\beta$ in diesem Bereich nichts Abnormes erkennen und pulsierte in normaler Weise. Nur an ihrem distalen Ende, entsprechend dem Bereich des 5. Lendenwirbels, schien sie verdickt und verklebt zu sein. Daher meißelte ich den Sakralkanal im Bereich des I. und 2. Sakralwirbels auf. Hier stieß ich nun auf einen fast wallnußgroßen harten Tumor, der vom 5. Lendenwirbel bis zum 2. Sakralwirbel reichte und einen Fortsatz in das rechte $\mathrm{I}$. Intervertebralloch sandte. Der Tumor saß deutlich intradural, war aber nach rechts hin durch die Dura durchgebrochen und ins I. Intervertebralloch und das Kreuzbein gewuchert. Mit der Dura war der Tumor ziemlich fest verwachsen. Bei Eröffnung der Dura, entsprechend dem caudalen Ende des Duralsackes, entleerte sich an einer Stelle etwas Liquor cerebrospinalis. Eine Kommunikation mit dem übrigen Duralsack bestand nicht. Die linke Hälfte des Tumors ließ sich stumpf von der Dura abtrennen und war hart, höckerig, von grauweißem fibromatösem Aussehen, der rechte Teil weicher, mehr graurötlich und diffus in die Dura und die Umgebung gewuchert, so daß er sich nicht von der Dura abtrennen ließ und ein Stück der letzteren reseziert werden mußte. Man hatte den Eindruck, daß links der Tumor seinen ursprünglichen gutartigen Charakter bewahrt hatte, während er rechts sarkomatös entartet war und den Knochen usuriert hatte. Es gelang mit einiger Mühe den Tumor auszulösen und im Zusammenhang mit seinem Fortsatz im Intervertebralloch herauszuheben. Dabei wurde der Durasack proximalwärts nicht eröffnet, obgleich die Dura etwa $I \mathrm{~cm}$ weit verdickt und verklebt zu sein schien.

Die Möglichkeit, daß in diesem Teil der Dura auch noch Tumorteile stecken können, kann trotz der guten Abgrenzbarkeit des Tumors 
nach dieser Richtung hin nicht geleugnet werden, doch schien mir in Anbetracht der Durchwachsung des Knochens, die die Aussicht auf eine dauernde Heilung gering erscheinen ließ, ein weiteres Suchen und eine breite Eröffnung der Dura in diesem Falle nicht indiziert.

Nach Auskratzung des usurierten Knochens und des Intervertebralloches wurde die Wunde durch tiefgreifende Nähte geschlossen, nur in der Mitte ein dünnes Zigarettendrain eingeführt.

Der Blutverlust während der Operation war ein relativ geringer, und Pat. überstand den Eingriff gut. Die Wunde heilte glatt bis auf die Stelle, an der das Drain gelegen hatte, doch ist auch diese Stelle jetzt (Ende Oktober I909), bis auf eine minimale, ganz oberflächlich granulierenden Wundfläche geschlossen.

Der exstirpierte Tumor erwies sich nach der Untersuchung im pathologischen Institut (Dr. Til p) als ein Fibros a rkom, das teils vorwiegend fibromatösen, teils vorwiegend sarkomatösen Bau zeigte. Stellenweise fanden sich Knochenzellen und kleine Inseln neugebildeten Knochens in demselben.

Bezüglich des sonstigen Heilungsverlaufes sei nur bemerkt, daß schon am Tage der Operation die ständigen Schmerzen im Kreuz und in den Beinen, über die Pat. seit 8 Jahren (!) klagte, aufhörten. Wenige Tage nach der Operation verschwand die Bewegungsbehinderung in den Beinen. Pat. konnte dieselben im Bett gut bewegen. Nach Io bis I2 Tagen lieB sich eine deutliche Einengung des anästhetischen Bezirkes, besonders links, nachweisen. Nach I6 Tagen konnte Pat. ihren Stuhlgang zurückhalten und spontan entleeren. Nach I8 Tagen fing sie an zu gehen, wenn auch schwankend und unsicher, so doch mit normalen großen Schritten fast ohne Unterstützung und ohne Schmerzen.

Sie kann jetzt - nach 5 Wochen - auch völlig schmerzfrei sitzen und seit dem 2r. Tage wieder spontan urinieren.1)

Der Fall beansprucht ein besonderes Interesse, weil das Herauswachsen eines Rückenmarkstumors durch ein Intervertebralloch zu den Seltenheiten gehört.

So weit mir bekannt, sind bis jetzt nur 7 solche Fälle beschrieben. Die Auffassung bezüglich des Ursprungs dieser Tumoren ist dabei bei den verschiedenen Autoren eine durchaus differente. Während B o e r n e r sich bei diesen Fällen mit Bestimmtheit für ein Einwachsen des Tumors von außen in den Wirbelkanal ausspricht, treten Bing und Bircher u. a. ebenso entschieden für den umgekehrten Modus ein. Ich glaube daß unser Fall keinen Zweifel darüber zuläßt, daß der Tumor

I). Io Tage später war auch die Reithosenanasthesie völlig verschwunden. 
intradural entstanden ist und wohl lange intradural bestanden hat, bis er dann die Dura durchbrochen hat und durch das Intervertebralloch nach außen gewuchert ist.

Nach den bisherigen Erfahrungen auf dem Gebiete der Rückenmarkschirurgie nehmen die Tumoren des Lendenmarks und der Cauda equina, wie besonders $\mathrm{C}$ a s s i r e r in seinem Referat über „Die Behandlung der Erkrankungen der Cauda equina“ betont, den letzten Platz bezüglich der dauernden Heilerfolge ein. Nach Stursberg sind bis jetzt im ganzen II 8 Fälle von Rückenmarkstumoren operiert worden mit einer Gesamtheilungszahl von ca. 35 Proz. Davon entfallen auf

die Halswirbelsäule 25 Fälle mit $44 \%$ Besserg., $36 \%$ Heilg.

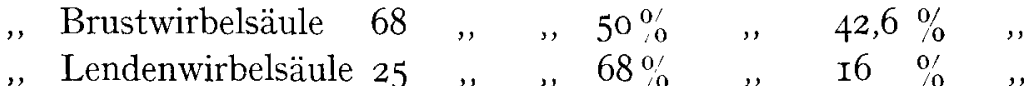
+ Cauda equina

Während die schlechten Resultate bei der Operation von Tumoren der Halswirbelsäule auf die Nähe der Medulla oblongata, die nur zu leicht in Mitleidenschaft gezogen wird, zurückzuführen sind, ist es bei den Tumoren der Lendenwirbelsäule in erster Linie der maligne Charakter der Neubildungen, der die Resultate hier so ungünstig gestaltet. So fand Cassirer, daß unter 5I Fällen, die er aus der Literatur zusammenstellte, höchstens in 6-8 Fällen (= I5 Proz.) Chancen für eine völlige Exstirpation des Tumors vorhanden waren, und nach seiner Berechnung erreicht der Prozentsatz der bisher erreichten Dauerheilungen für die Tumoren der Cauda equina noch nicht Io Proz.

Abgesehen von der Malignität der Tumoren sind es die ungünstigen Wundheilungsverhältnisse, die den Erfolg in diesen Fällen erschweren. Um Zugang zum Lendenmark und zur Cauda equina zu erlangen, muß man große, tiefe Wunden anlegen, die oft sehr stark bluten und in denen es leicht zu Infektionen kommt. Letztere können um so eher eintreten, als die Pat. Urin und Stuhl unter sich lassen und nur mit Mühe trocken gehalten werden können. Bei Anwendung des Dauerkatheters kommt es oft zu schweren Cystitiden, die zu aufsteigenden Pyelonephritiden führen. Die Pat. neigen außerordentlich $\mathrm{zu}$ schwerem Decubitus mit seinen gefährlichen Folgen. Endlich macht das Auffinden des Tumors, 
der gerade in diesem Abschnitt der Wirbelsäule oft sehr schwer genau auf einen bestimmten Wirbelbereich zu lokalisieren ist, manchmal große Schwierigkeiten.

Der Chirurg hat nun, durch die Ausbildung der Technik und immer erweiterter Erfahrung, mancherlei Hilfsmittel im Kampf gegen diese Schwierigkeiten. Seitdem wir wissen, daß den Pat. durch Fortnahme selbst von $6-7$ Wirbelbögen kein dauernder Schaden erwächst, können wir schnell und doch relativ schonend ausgiebigen Uberblick über die vorliegenden Verhältnisse gewinnen. Durch die prinzipielle Eröfnung der Dura in allen Fällen, in denen sich kein extraduraler Tumor findet, wird mancher Tumor gefunden werden, der noch bis vor kurzem der eingreifenden Hand entging. Durch die Schaffung glatter Wundverhältnisse, speziell durch Verzicht auf die temporäre Wirbelresektion nach $\mathrm{Urban}$, die, wie in dem Fall von $\mathrm{S}$ o $\mathrm{n} \mathrm{n}$ e $\mathrm{n}$ burg, nur zu leicht zu Necrosen und sekundärer Meningitis führt, wird nach Möglichkeit der Infektion vorgebeugt. Durch schonende Anwendung des Dauerkatheters wird gleichfalls die Infektion, gleichzeitig aber auch die Entstehung des Decubitus bekämpft. Endlich scheint nach den Erfahrungen amerikanischer Chirurgen (Cushing, Crile) im Urstropin ein wirksames Mittel gefunden zu sein, um meningeale Infektionen zu bekämpfen oder ihnen vorzubeugen, da sich dasselbe sehr bald nach interner Verabreichung in ziemlich großen Mengen als Formalin im Liquor cerebrospinalis nachweisen läßt.

Nur gegen die Malignität der Tumoren sind wir machtlos, da auch bei ausgiebigsten Exstirpationen fast stets das tötliche Rezidiv folgte.

Immerhin schafft auch in diesen Fällen die Operation wesentliche Besserung. Nicht nur wird für eine gewisse Zeit das Rückenmark vom Druck des Tumors befreit und werden damit in vielen Fällen die spinalen Funktionen völlig wiederhergestellt, sondern es kann durch Entfernung der knöchernen Umwallung auch eine dauernde Druckentlastung des Marks im Falle des Rezidivs, analog den Vorgängen nach Ventiltrepanationen bei inoperablen Hirntumoren bedingt werden. Dadurch sind in einer Reihe von Fällen die Pat. dauernd von Störungen seitens des Rückenmarks nach der Operation befreit geblieben, trotzdem der Tumor rezi- 
582 Rosenfeld u. Guleke, Über einen Fall von oper. Tumor der Cauda equina.

divirte und schließlich den Tod des Pat. herbeiführte. - Für die Indikationsstellung zur Operation speziell bei den das Lendenmark und die Cauda equina komprimierenden Tumoren kommt noch in Betracht, da $B$ diese Tumoren nach den bisherigen Erfahrungen vielfach von den Wirbeln ausgegangen sind, so daß zu ihrer Entfernung eine Eröffnung der Dura mit allen damit verknüpften Gefahren nicht notwendig war. Dementsprechend betrug auch der Prozentsatz des postoperativen Exitus nach Stursberg bei diesen Operationen nur I6 Proz. (gegenüber 26,4 Proz. beim Brustmark und 40 Proz. beim Halsmark). Der Nutzeffekt der Operation bezüglich der Wiederherstellung der Rückenmarksfunktionen war ein verhältnismäBig sehr günstiger $=68$ Proz. wesentliche Besserung. Auch hiernach ist also in Fällen von malignen Tumoren, bei denen eine Dauerheilung nicht zu erwarten ist, der Eingriff doch als durchaus gerechtfertigt anzusehen;

Auf Grund dieser Erfahrungen sind wir, wie Stu rs berg, $\mathrm{B}$ i n $\mathrm{g}$ und B ir c h e r u. a. betonen, in allen Fällen von diagnostizierten und lokalisierten Rückenmarkstumoren nicht nur berechtigt, sondern verpflichtet operativ vorzugehen und mit Recht wird für zweifelhafte Fälle in neuester Zeit die Probelaminektomie sowohl von Chirurgen, als auch von Neurologen gefordert.

Auch in unserem Falle ist es zweifelhaft, ob die Besserung, die durch die Operation erzielt ist, eine dauernde Heilung bedeutet oder nicht. Da der exstirpierte Tumor ein Fibrosarkom war, ist die Möglichkeit des Rezidivs eine große. Trotzdem wird aber bei dem Befunde, den die Pat. jetzt darbietet, wohl kein Zweifel darüber herrschen können, daß die Operation in solchen Fällen indiziert ist, um so mehr, als durch die Entlastung des Rückenmarks einer Wiederkehr der spinalen Störungen vielleicht vorgebeugt ist, selbst wenn ein Rezidiv auftreten sollte. 